\title{
Recurrent Thromboembolic Events in a Patient With Dysautonomia: A Case Report
}

\author{
Kyle C. Smith ${ }^{\mathrm{a}, \mathrm{c}}$, Andrew M. Freeman ${ }^{\mathrm{b}}$
}

\begin{abstract}
Recurrent unexplained thromboembolic events are managed by anti-coagulation medications with or without a known etiology. In patients with autonomic instability syndromes there has been a recent report of a link to anti-phospholipid syndrome (APS) but also the mechanism of dysautonomia itself may contribute to clot formation. In this case report we report the case of a 26-year-old female with postural tachycardia syndrome (POTS) and gastroparesis who suffered chronic thromboembolic phenomenon diagnosed after an episode of amaurosis fugax. This patient's case will serve to highlight the importance of recognizing risk factors for blood clots in patients with dysautonomias.
\end{abstract}

Keywords: Dysautonomia; Postural tachycardia syndrome; Gastroparesis; Thromboemboli; Clots

\section{Introduction}

A patient with a history of autonomic dysfunction, postural tachycardia syndrome (POTS) diagnosed by tilt table study and gastroparesis, developed sudden onset unilateral blindness diagnosed as a transient ischemic attack (TIA). Further evaluation with a retina examination showed evidence of chronic recurrent thromboembolic events. POTS is a disorder characterized by a fall in blood pressure when changing position from a seated to standing position that produces

\footnotetext{
Manuscript accepted for publication May 15, 2014

${ }^{a}$ Kansas City University of Medicine and Biosciences, 1750 Independence Blvd, Kansas City, MO 64106, USA

${ }^{\mathrm{b}}$ Department of Medicine, Division of Cardiology, National Jewish Health, 1400 Jackson St. Rm J307, Denver, CO 80206, USA

${ }^{\mathrm{c}}$ Correponding Author: Kyle C. Smith, Kansas City University of Medicine and Biosciences, 1750 Independence Blvd, Kansas City, MO 64106, USA. Email: kysmith14@gmail.com

doi: http://dx.doi.org/10.14740/jmc1806w
}

symptoms of cerebral hypoperfusion - weakness, lightheadedness, blurry vision and/or loss of consciousness [1]. Gastroparesis is delayed gastric emptying due to a discordant, sympathetic and parasympathetic nervous system that produces nausea, vomiting, early satiety, bloating and/or upper abdominal pain [2]. The normal course of these disorders is usually mitigated by life-style modification and appropriate medications, and patients do not usually suffer life-threatening sequelae as a result.

\section{Case Report}

A 26-year-old Caucasian female with a history of supraventricular tachycardia and superficial venous thrombosis and family history of maternal pulmonary embolism, presents to the emergency department (ED) with bloody diarrhea, abdominal pain, nausea, vomiting and orthostatic hypotension diagnosed as ischemic colitis. Subsequently she developed early satiety, nausea and vomiting which was diagnosed as gastroparesis with a gastric emptying study.

Months later she developed sudden onset blindness in her left eye that was diagnosed as a TIA. Her retinal examination showed evidence of on-going and chronic thromboembolic phenomenon. She was started on Coumadin.

She presented to the cardiologist after these events with recurrent near syncope, chest pain and marked fatigue. The patient underwent a series of tests. An EKG showed sinus bradycardia at 53 beats per minute (bpm) with nonspecific T-wave and repolarization abnormalities. Echocardiogram revealed a patent foramen ovale (PFO), but was otherwise unremarkable with normal left ventricular function. A Holter monitor showed an average heart rate of 79 , one premature ventricular contraction and five premature atrial contractions. The tilt table testing confirmed the diagnosis of POTS with a persistent increase in heart rate greater than $30 \mathrm{bpm}$ within 10 min of postural change, and associated with symptoms including dizziness, shortness of breath and palpitations.

Consideration was given to PFO closure, but recent data [3] and the multi-factorial causes for her neurologic and opthamalogic symptoms suggested anti-coagulation would be the best initial approach. 
Hematologic panels were performed that showed the lupus anticoagulant (LA) was sensitive. Lupus-like inhibitors (LLI), Russell viper venom, B2 glycoprotein, cardiolipin $\mathrm{IgG}, \operatorname{IgM}$ and $\operatorname{IgA}$ are all negative. Therefore this patient does not meet the laboratory portion of the Sapporo criteria [4] for definite anti-phospholipid syndrome (APS).

The patient started cardiac rehabilitation and demonstrated overall improvement in her symptoms, but did have one episode of presyncope that prompted a visit to the ED. She was given two bags of intravenous fluids and symptoms improved.

At an office visit almost 1 year later, the patient reported a recent episode of acute onset tinnitus, headache and blurred vision that prompted an ED visit. Work-up that included a head CT was negative and the patient was subsequently discharged.

Currently the patient is being managed with medications for her clotting history and gastroparesis and life-style modifications like maintaining hydration and an exercise program for POTS, with decent success. She has been able to reduce the frequency of her syncopal and near-syncopal events and has suffered no further vision changes or TIA symptoms.

\section{Discussion}

Autonomic dysfunction in conjunction with thrombosis has been described in animal studies. Cats that underwent vagus nerve stimulation developed a hyper-coagulable state [5]. Additionally, a recently published study proposes an association between POTS, neurocardiogenic syncope, and orthostatic hypotension and APS [6], leading the author to argue that POTS patients with migraines, memory loss, balance trouble, livedo reticularis, Raynaud's, history of miscarriage or family history of blood clots should all be tested for APS. Additionally, the underlying mechanism of autonomic instability that includes venous pooling could itself increase a person's risk for thrombosis as a component of Virchow's triad [7].

\section{Conclusion}

This patient presents with a unique constellation of autonomic instability and thromboembolism. The consequences of even a single thromboembolic event can lead to morbidity and even mortality, and therefore it is important to recognize patients who are at increased risk for developing clots.
If autonomic abnormalities can predispose patients to these events, it is important for the treating physician to be aware of the connection between these two conditions. In addition, the risks should be explained to every patient, but especially in those at a particular risk, such as this patient - a young white female with a family history of pulmonary emboli and who was on oral contraceptive pills.

\section{Competing Interests}

The authors declare that they have no competing interests.

\section{Author Contributions}

KS analyzed and interpreted the patient data and wrote the manuscript. AF is the treating physician and contributed to the writing the manuscript. All authors read and approved the final manuscript.

\section{References}

1. Jacob G, Biaggioni I. Idiopathic orthostatic intolerance and postural tachycardia syndromes. Am J Med Sci. 1999;317(2):88-101.

2. Camilleri M, Parkman HP, Shafi MA, Abell TL, Gerson L, American College of G. Clinical guideline: management of gastroparesis. Am J Gastroenterol. 2013;108(1):18-37; quiz 38.

3. Larrue V, Massabuau P. Patent foramen ovale and ischemic stroke. Minerva Med. 2012;103(3):199-207.

4. Miyakis S, Lockshin MD, Atsumi T, Branch DW, Brey RL, Cervera R, Derksen RH, et al. International consensus statement on an update of the classification criteria for definite antiphospholipid syndrome (APS). J Thromb Haemost. 2006;4(2):295-306.

5. Kuznik BI, Mishchenko VP. Effect of vagus nerve stimulation on coagulability of the blood in cats. Bull Exp Biol Med. 1975;77(7):723-725.

6. Schofield J, Blitshteyn S, Shoenfeld Y, Hughes G. Postural tachycardia syndrome (POTS) and other autonomic disorders in antiphospholipid (Hughes) syndrome (APS). Lupus. 2014.

7. Bagot CN, Arya R. Virchow and his triad: a question of attribution. Br J Haematol. 2008;143(2):180-190. 